\title{
Postharvest bitter pit detection and progression evaluation in 'Honeycrisp' apples using computed tomography images
}

Sanaz Jarolmasjed ${ }^{1, \dagger}$, Carlos Zúñiga Espinoza ${ }^{1, \dagger}$, Sindhuja Sankaran ${ }^{1,2}$, Lav R. Khot ${ }^{1,2 *}$

${ }^{1}$ Department of Biological Systems Engineering, Washington State University, Pullman, WA

${ }^{2}$ Center for Precision and Automated Agricultural Systems, Department of Biological Systems

Engineering, IAREC, Washington State University, Prosser, WA

† Equal contributing authors

* Corresponding author: Lav R. Khot; Email: lav.khot@wsu.edu; Phone: 509-786-9302 9

Abstract

Bitter pit is a physiological disorder that is defined as brown, corky and roundish lesions, which can develop in apples before and after harvest. This disorder greatly reduces the product utilization value of the fruit, and can result in several million dollar economic loss to the apple industry. Computed Tomography (CT) imaging is a non-destructive and rapid sensing technique that can be applied to packaged apples. In this study, healthy and bitter pit Honeycrisp apples were harvested from two field sites and stored for 63 days. CT images of the sampled apples were collected on $0,7,14,21,35$ and 63 days after harvest. Images were analyzed to estimate the total pit area in each of the individual apples and were related to pit incidence and progression in different stages of storage. Results showed pit development during the storage period in bitter pitted apples. The rate of progression differed in samples collected from different field sites. Further analysis for pit distribution along each of the bitter pit affected apples showed $54 \%$ of pits located at the calyx-end of apples in comparison with middle and stem-end.

Classification of healthy and bitter pitted apples using logistic regression based method resulted in false negative of $7-21 \%$. 
Keywords: Image processing; Classification; Apple storage; Non-invasive sensing.

\section{1. Introduction}

Washington State produced about 5,100 million kg of apples in 2014 (U.S. Department of Agriculture- National Agricultural Statistics Service, USDA-NASS, 2015). Washington State apple accounts to about $60 \%$ of the total U.S. production, significant quantity of which is exported. As most of the produce is for fresh market, size and quality of the fruits are two key factors that govern the market value. Therefore, fruit inspection and sorting by human and automated imaging systems on the packaging lines are critical while packing premium quality fruits such as Honeycrisp without any defects. In case of apples, defects and disorders include arthropod damage (codling moth, mealybugs, aphid damage), postharvest diseases (blue mold, gray mold, mildew), physiological disorders (bitter pit, superficial scald, core flush), mechanical and field injuries (bruising, frost, hail damage), and fruit finish (sunburns, Jones et al., 2007).

Researchers have integrated and developed sensing systems for detecting diseases hyperspectral, fluorescence, near-infrared, X-ray imaging, and visible-near infrared spectroscopy. Bennedsen et al. (2005) used near-infrared imaging to identify surface defects on eight different varieties of apples. A high spatial resolution camera was used to collect six images from a fruit sample that was rotated. Two filters of 740 and $950 \mathrm{~nm}$ were used to capture dark marks, bruises, and faint marks on the sample images. This system showed 78 to $92.7 \%$ accuracy in finding total defective area. Similarly, Xiao-bo et al. (2010) used three color chargecoupled device (CCD) camera to detect apple defects with nine images from each apple. The

47 number of defects such as bruising, scab, fungal growth, and diseases along with stem-end and 
features were identified. The stem-end and calyx would account to two anomalies (identified as defects) on surface that would appear on all apple images. Their system achieved classification precision of $95.8 \%$. The classification error increased from $4.2 \%$ to $21.8 \%$ when one camera was used instead of three as more false positive samples were observed.

Bitter pit (BP) is one of the key defects in certain apple cultivars (e.g. Honeycrisp) which is defined as a physiological disorder and characterized by a depression in the flesh of apples (Amarante et al., 2013). Bitter pit symptom begins from plasma membrane segregation in certain locations within the fruit, and the pits get dehydrated with tissues becoming corky and darker in color (Fuller, 1979). One of the hypothesis associated with bitter pit formation in fruits during production is the $\mathrm{Ca}^{+2}$ deficiency due to improper uptake of nutrients by the plant. The disorder has also been linked to differences in growing conditions such as site location, elevation, and high summer temperatures (Van der Boon, 1980; Gutiérrez, 2002). Few researches have reported trends of bitter pit progression in peel of the fruit throughout the time after harvest (Ferguson and Waltkins, 1989; Nicolaï et al., 2006). Rosenberger et al. (2001) studied bitter pit development up to 85 days storage period in Honeycrisp apples to evaluate the effect of calcium and fungicide applications in bitter pit development. Only one of three trials showed the effectiveness of calcium and fungicide treatment in $40 \%$ reduction in bitter pit incidence compared to control trees, which could be associated with orchard locations and other 67 conditions. One of the biggest challenges in bitter pit detection is that the pit formation starts internally and pits appear on the fruit surface with time (possibly months after harvest). Fruits appear healthy during harvest and packaging but develop symptoms during storage, which makes the produce unmarketable in the fresh market. Bitter pit development in production and

72 during storage are reported to occur in up to $30-50 \%$ of the apples in cultivars susceptible to this 73 defect (Rosenberger et al., 2001; Amarante et al., 2006) and disorder severity varies with 
74 location and production year. If the internal bitter pit development can be detected using non-

75 invasive sensing technique that can non-destructively scan fruits from within, such fruits will not

76 be marketed. This process can save saving labor, packaging, transportation, and other

77 associated costs.

Fluorescence imaging has been used to detect bitter pit on 'Golden Delicious' apples

79 (Lötze et al., 2006). Although difference between averaged cumulative distribution functions (CDFs) of pitted and non-pitted apples was reported, fruit misclassification rate was about $50 \%$. In another study, hyperspectral near infrared imaging system was successfully used to identify visible and non-visible bitter pit lesions (Nicolaï et al., 2006). A line scan near infrared camera with a spectrograph was used to capture spectral image of pitted and non-pitted apples. This system was reported to be able to detect bitter pit lesions that were not visible to human eye. However, system failed to differentiate bitter pit and other types of disorders that created corky tissues. The techniques discussed above focused on surface and near surface apple bitter pit 87 detection. contact bitter pit detection technologies will lead to reduced costs associated with packaging

97 materials, labor and transportation, and improved marketability and economics of the fresh market produce. 
The X-ray CT can scan objects to generate two- (2D) and three-dimensional (3D)

100 images. Stacked series of CT image data can provide the physical and physiological structure of 101 the biological substances (Magwaza and Opara, 2014) via measuring X-ray absorption by the 102 sample. Thus, the CT imaging can display the internal structure of the sample, thereby serving 103 as a non-destructive tool to inspect the internal defect and disorders in fruits (Schatzk et al., 104 1997; Du and Sun, 2004; Lei et al., 2014). Herremans et al. (2014) successfully applied X-ray 105 CT to detect watercore disorder in apple, with accuracy of up to $89 \%$. CT images of the 106 watercore affected apples showed higher intensity due to increased density of the tissue. 107 Magwaza and Opara (2014) demonstrated the X-ray CT imaging capability to study the quantity 108 and distribution of edible and non-edible portions of pomegranate fruit for breeding purposes. 109 They created 3D images of scanned fruits and estimated volume occupied by aforementioned 110 portions. The X-ray CT has also been used in determining fresh chestnut internal quality (Donis111 González et al., 2014). Six images were extracted per chestnut including mean and maximum 112 intensity images from three different views ( $X Y$ plane, $X Z$ plane and $Y Z$ plane). The images 113 were then segmented using balanced histogram thresholding method and classified by 114 quadratic discriminant analysis (QDA) for quality class attributes. Classification accuracies were 115 between $86-96 \%$ depending on the number of classes which ranged between 2-5 classes. In 116 addition to quantification of internal microstructural changes in fruit tissues (Herremans et al., 117 2014), X-ray CT can also sample a large field of view with minimal sample preparation 118 (Magwaza and Opara, 2014). In line with above discussion, the overall objective of this study 119 was to evaluate applicability of X-ray CT-based imaging technique with robust image processing 120 algorithm for rapid and precise detection of external and internal bitter pit defects in Honeycrisp 121 apples. Such development and validation may assist in identification of bitter pit disorder on the 122 apple packaging lines for eliminating pitted fruits prior to packaging and transportation. 


\section{Materials and Methods}

125

\subsection{Field Sampling}

Honeycrisp apples were harvested at commercial maturity from two different orchard locations, Prescott, WA (field site-1) and Burbank, WA (field site-2). Field site-1 has an elevation of $365 \mathrm{~m}$, maximum average temperature of $32{ }^{\circ} \mathrm{C}$, minimum average temperature of $14{ }^{\circ} \mathrm{C}$, and $35 \%$ average relative humidity $(\mathrm{RH})$. Corresponding characteristics for field site-2 were $114 \mathrm{~m}$ elevation, $31^{\circ} \mathrm{C}$ and $14^{\circ} \mathrm{C}$ maximum and minimum average temperature, and $35 \%$ average $\mathrm{RH}$. In both sites, the apples were sampled from eight-year-old trees having M9 rootstock and canopies planted with 'V' trellis system. Apples were harvested on August 29, 2014 and were immediately transported to Pullman, WA and were stored in cold storage at $5^{\circ} \mathrm{C}$. Samples consisted of 20 healthy $(\mathrm{H})$ and 20 bitter pit-affected (BP) apples from each field site. The BP apples were visually symptomatic. Reason for selecting BP apples with visual symptoms was because previous literature (Nicolaï et al., 2006) had indicated challenges in prediction of BP development in healthy apples, leading to unbalanced sample size. To avoid such scenario, healthy (with potential of BP development) and BP (symptomatic) samples were selected. Moreover, the BP samples would also aid in understanding the BP progression during storage. Apples were wiped clean and sorted in a separate packaging box per fruit class, simulating commercial packaging conditions. Additionally, apples were numbered individually for tracking throughout the 63 days after harvest (DAH) study period. No additional treatments were applied to the fruits during storage.

\subsection{Data Acquisition}

During storage, fruits from field site- 1 and -2 were scanned using CT imaging technique on $0,7,14,21,35$ and 63 DAH. CT imaging was performed with 16-slice CT scanner (Aquilion ${ }^{\circledR}$, 
Toshiba, Shimoishigami, Tochigi-ken, Japan) located at Diagnostic Imaging (Radiology) Lab, Veterinary Teaching Hospital, Washington State University, Pullman, WA. The voltage and current requirement of the device were $120 \mathrm{kV}$ and $150 \mathrm{~mA}$, respectively. For CT imaging, fruits were stacked in two boxes, one per field site. In each box, two layers of fruits were placed, with

15220 bitter pit affected fruits on bottom layer and 20 healthy fruits on top layer. Typical sample box 153 orientation during a CT scan was as depicted in Fig. 1. During the imaging process, images 154 were taken from three different directions: longitudinal, transversal, and sagittal. The number of 155 scans per box were equal with $3 \mathrm{~mm}$ resolution between scan layers.

\subsection{Image Analysis}

CT image datasets (a dataset/scan day/field location) were individually analyzed using custom algorithms developed in Matlab ${ }^{\circledR}$ using Image Processing Toolbox (Mathworks, Natick, MA). Overall, the absorption of X-ray beam by the fruit samples can be estimated in terms of CT number, a number that relates the X-ray absorption by the sample compared to that of absorption by water. The CT number data can then be used to create a gray scale image of the sample (Salmanizadeh et al., 2015). The CT number data represent pixel values in gray scale image. In this study, the gray scale images of transversal view were further processed to extract bitter pit features that indicated differences between healthy and bitter pit fruits.

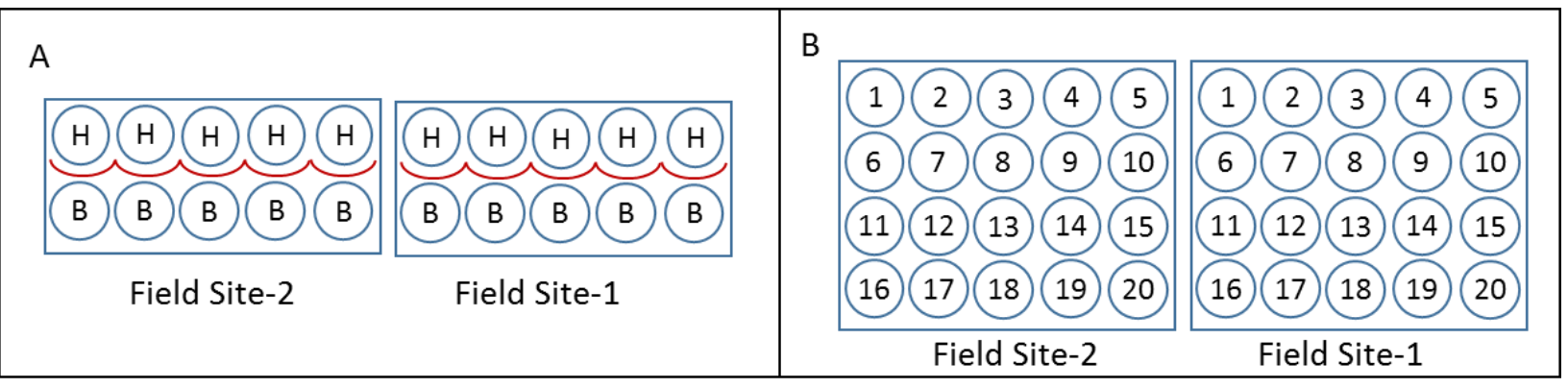


Fig. 1. Schematic depicting boxed apple samples for CT based imaging; (A) longitudinal view of the boxes imaged with ' $\mathrm{H}$ ' and ' $\mathrm{B}$ ' representing healthy and bitter pit affected apple layers, and (B) transversal view of the boxes before imaging with samples numbered from top-left to bottom-right in increasing order for referencing.

Image processing involved the following steps. First, the central CT image with maximum diameter of apples in the fruit layer within the sample box was manually selected to establish a 'bounding box model' for identification and registration of individual apples in the box. This bounding box is a rectangle boundary placed around every apple in the selected central CT image. In this way, it was possible to identify and index each apple with a distinctive number, and determine properties such as area, perimeter, or centroid. In parallel, a stack of cross-sectional CT images was created, along with central CT image. The aforementioned bounding box selection from the central CT image was applied to all of the cross-sectional CT images for fruit delineation (registration/indexing). Fruits were then uniformly numbered following bounding box pattern from central CT image. Individual fruit images were segmented after transforming grayscale CT images into the binary images. In the binary image, apple flesh was observed as white (1). Meanwhile, the pits and core inside the fruit along with the image background was observed as black (0). Small objects or pieces of surrounding fruits were eliminated and only the fruit image in the bounding box was acquired. Also, morphological operations (watershed, erode, and dilate) were applied for better segmentation of the images. All the mentioned processes, except the selection of the central CT image, were performed by the developed algorithm.

After segmenting individual fruits in CT image layers, two types of data were extracted. In step one, pits and cores of images were eliminated (converted to 1) to extract apples without pits (AWOP) and in the second step, core only sections were eliminated to extract apples with pits (AWP). Apple core radius was defined as one-fifth of the maximum radius measured from 
192 the centroid. The pits were defined as objects with an area $<150$ pixels. This threshold was 193 optimized based on visual comparison and used in processing all of the samples. The overall 194 goal of the above steps was to determine the ratio between pits and the total area per fruit 195 termed as pit ratio (PR) using the following equation:

$196 \quad \mathrm{PR}=\sum_{i=1}^{n}\left(\frac{\mathrm{AWOP}-\mathrm{AWP}}{\mathrm{AWOP}}\right)_{i}$

197 where, $\mathrm{n}$ refers to the total number of apple image layers.

198 Fig. 2 outlines the chronology of steps followed to obtain the PR. The PR data were used to 199 estimate bitter pit area per fruit and pit progression during storage. For a given DAH condition, 200 PR per fruit was compared to estimate the disorder progression. Above described analysis 201 steps were repeated for $0,7,14,21,35$ and 63 DAH storage datasets. 


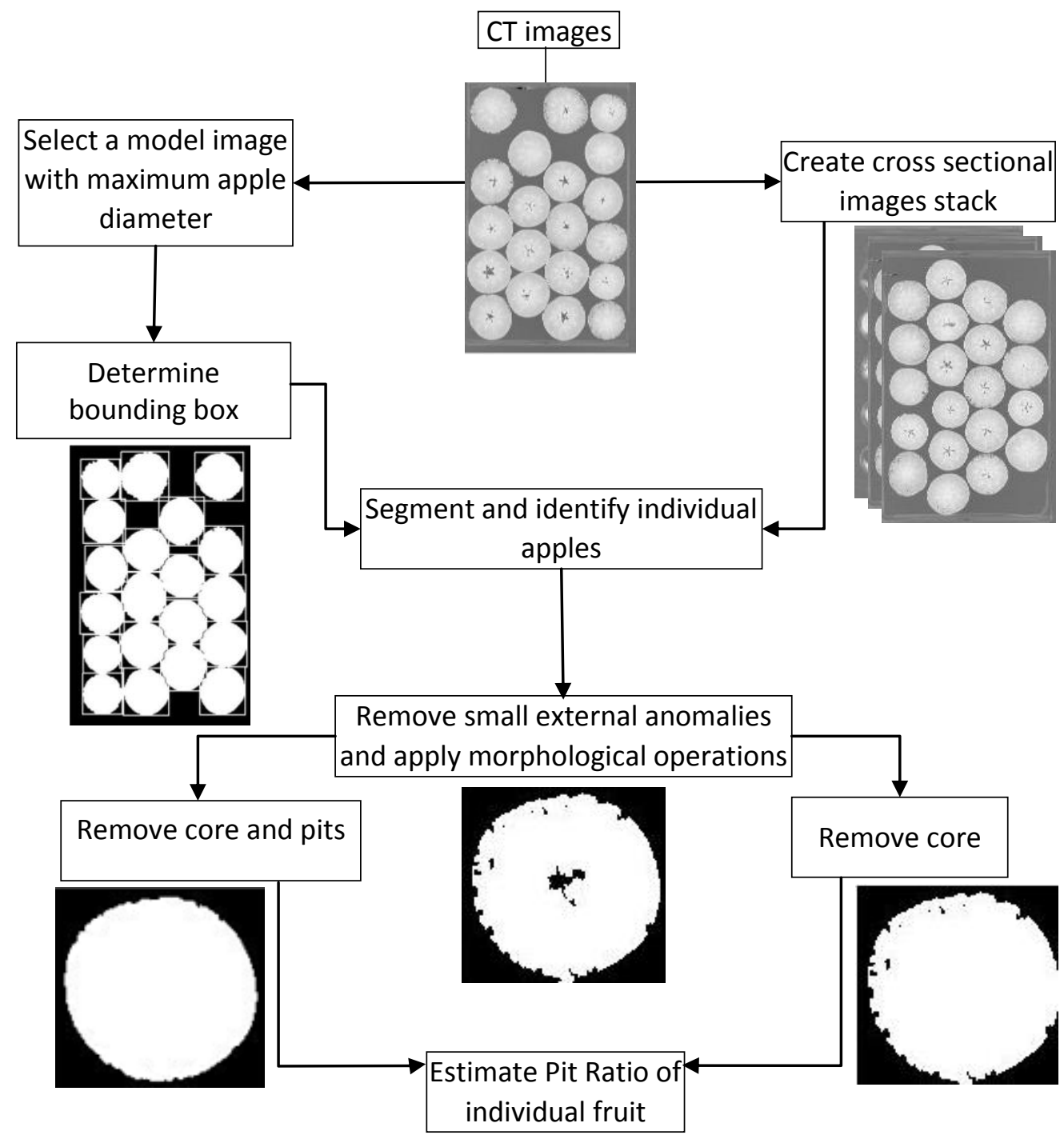

Fig. 2. CT image dataset processing steps implemented in the developed algorithm to estimate bitter pit ratio per sample and overall disorder progression during $63 \mathrm{DAH}$ storage period. monitored by extracting PR progression (PP) to estimate percentage increase of pit incidence.

207 PP was calculated for each sample by comparing the current PR estimate to that of the previous 208 one (Equation 2). 
210 where, $P R_{i}$ is sum of the Pit Ratio for any of the DAH for a specific apple, and $P R_{n}$ is sum of pit

211 ratio before $i$. As an example, to compare the bitter pit progression of an individual apple from

21235 to 63 days, $n$ and $i$ will represent $35 \mathrm{DAH}$ and $63 \mathrm{DAH}$ data, respectively. This comparison

213 shows the increased amount of the bitter pit area in each fruit during 63 days storage.

Bitter pit distribution in each apple was also determined through additional PR analysis.

215 Apples were divided in three sections representing stem-end (top), middle, and calyx-end

216 (bottom). Each section covered 20 to $30 \mathrm{~mm}$ of the apple vertical cross-section area. PR was

217 added in each of the three sections and percentage of pits with respect to the total apple depth

218 (vertical) was estimated.

\subsection{Healthy and Bitter Pit Fruit Classification}

Apples were classified as healthy and bitter pit apples using pit ratio dataset. Logistic regression was used to determine the class threshold. It uses logit as core mathematical method for discrimination of the classes by describing a categorical outcome from a continuous

224 or numerical independent input variable. In this study, model demonstrated effect of continuous 225 independent variable (PR) on the dichotomous dependent variable, i.e. healthy or bitter pit 226 class. The class method uses maximum likelihood estimation to fit the model to the data 227 (Trexler et al., 1993). The logistic regression algorithm develops points in parallel corresponding 228 to PR values (x-axis) for two classes in order to categorize each sample. The logistic regression 229 creates a S-shaped curve predicting the logit of the variable based on continuous predictor (PR 230 value). The model outcome is dichotomous and the line describes the regression curve on both 231 ends, as logit transformation is applied to dependent variable (Peng et al., 2010).

\subsection{Statistical Analysis}


Descriptive and multivariate statistical analysis was performed using R Studio (ver.

0.99.451, R Studio Inc., Boston, MA). Results were inferred at 5\% level of significance.

\section{Results and Discussion}

\subsection{Bitter Pit Development and Progression Monitoring}

After determining PR of each fruit using the intensity variations captured by CT between healthy and bitter pit affected tissues in the samples, it was possible to obtain pit development throughout $63 \mathrm{DAH}$ storage period. Figure 3 displays the PR trend for samples from field sites-1

242 and -2. For field site-1 (Fig. 3A), PR in bitter pit affected apples showed increasing trend up to 24363 days in storage. In contrast, PR and associated variation in healthy apples throughout the 244 study period was close to zero. Increase in PR bitter pit progression during 63 DAH storage 245 period was significant for the individual locations. Similar trend was observed for bitter pit 246 affected fruits sampled from the field site-2 (Fig. 3B). Although not significantly different, field 247 site-2 sampled fruits showed lower rate of bitter pit progression. Differences in the production 248 management, climatic variation, and levels of fruit maturity at the harvest might have been 249 attributed for such variations, as reported by Van der Boon (1980) and Gutiérrez (2002).

250 However, CT data of samples from both sites confirmed that the bitter pit disorder tend to 251 progress during the storage.

A

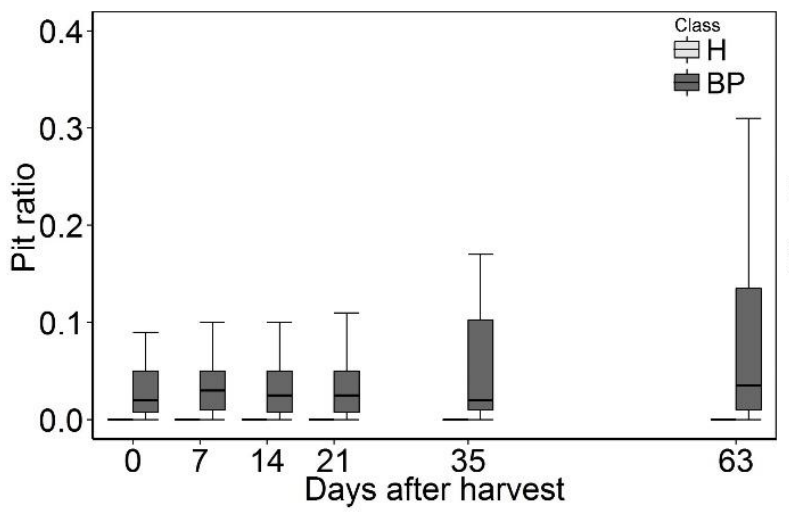

B

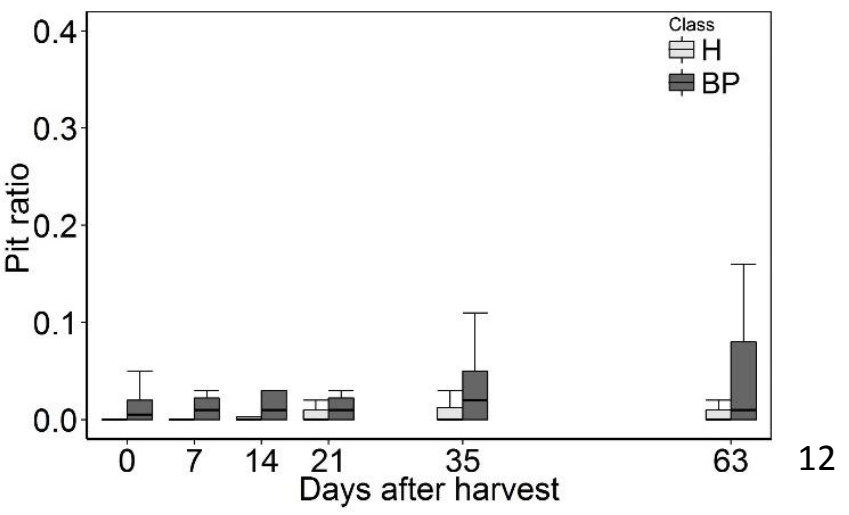


258 Fig. 3. Temporal pattern of bitter pit development in healthy and bitter pit affected 'Honeycrisp' apples collected from field sites-1 (A) and $-2(B)$ as monitored using CT imaging during $63 \mathrm{DAH}$ storage period. In boxplot, the upper and lower whisker represent the maximum and minimum

261 pit ratio values, upper and lower box borders represent the $75^{\text {th }}$ and $25^{\text {th }}$ percentile values, respectively, and the horizontal dark line indicates the median.

In the field site-2, healthy fruits (samples) showed PR from CT images to predominantly increase at $21 \mathrm{DAH}$, which remained stable up to $63 \mathrm{DAH}$. Further evaluation of healthy samples confirmed that PR increase could be associated with fruit bruising injury caused at harvest. Above results confirm the suitability of CT based imaging not only in capturing the bitter pit disorder but also for other bruising and handling injuries in the fruit. This also highlights the challenge in developing the image processing algorithm that can segregate bitter pit disorder

270 from other fruit injuries. Overall, algorithms developed in this study can effectively identify 271 internal pits and defects in addition to the incidences of pits in cuticle surface layer.

272 Besides PR analysis, the bitter pit progression data were used to estimate pit incidence 273 in the apples. Data showed an increase in the bitter pit percentage during 63 days of storage. A 274 few representative sample images with their PR and PP data are presented in Figure 4 and 275 Table 1. Each row is showing a specific apple layer in three different storage stages that display 276 the bitter pit progression with storage time. A significant development of pits was observed in 277 the fruit cuticle and underneath during 63 days of storage. In general, bitter pit progression in 278 individual bitter pit affected apples increased from 0.1 to 7 times from the day of the harvest to 279 the $63 \mathrm{DAH}$. 
282 injuries other than bitter pit. One of the limitations of the algorithm developed in this study is that 283 the Pit Ratio includes other types of damages in the fruit if any. Further improvements to the 284 algorithm are being conducted to differentiate various types of damages from bitter pit disorder 285 using shape-based segmentation of fruit image areas. Improved algorithm can be used not only 286 in detecting fruit disorders and damages, but also to quantify bitter pit occurrence in plant 287 breeding programs while developing new bitter pit resistant cultivars. 
Day-0
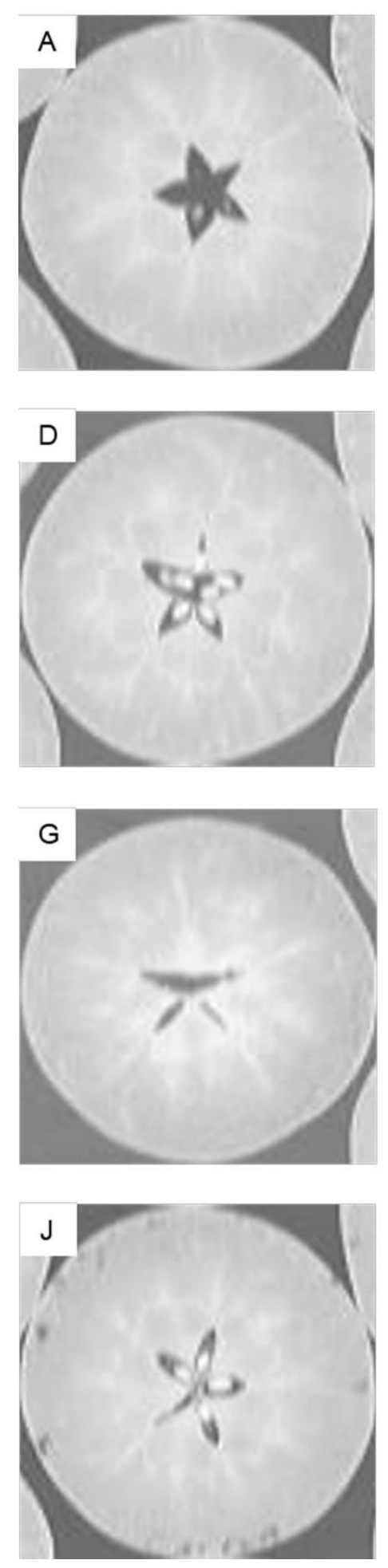

Day-35
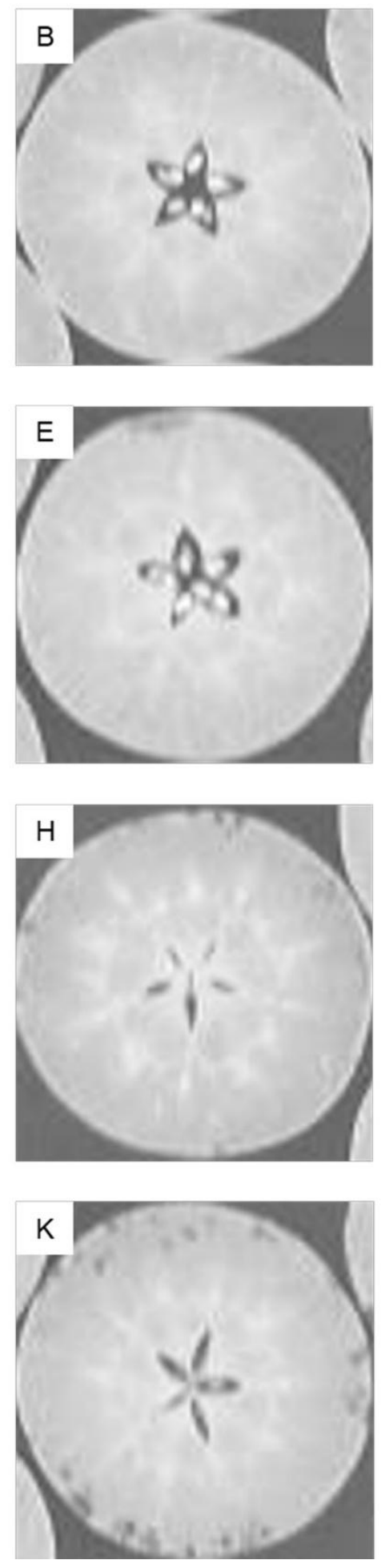

Day-63
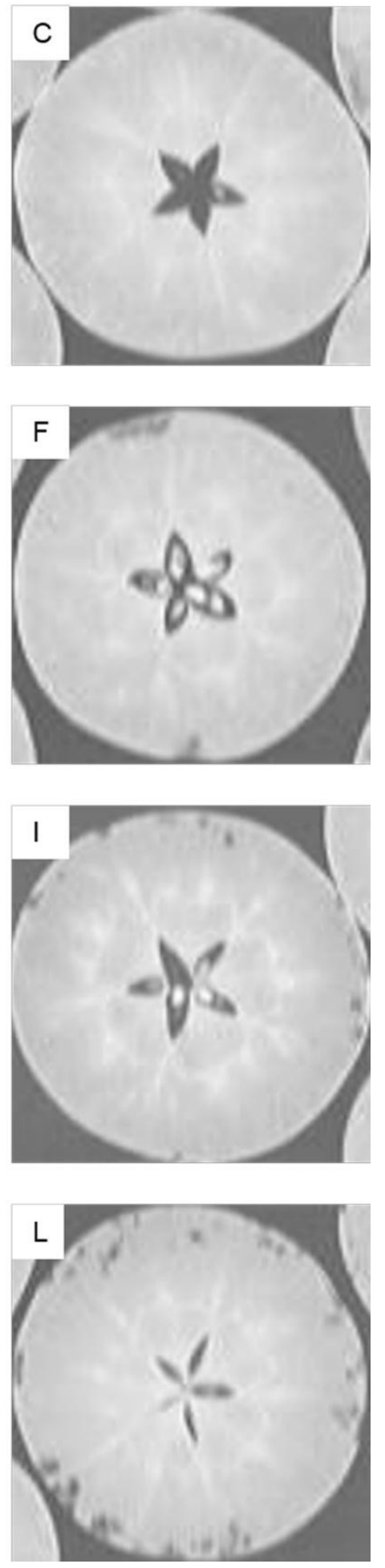

Fig. 4. CT images of healthy apple without bruise (A-C), healthy apple with bruise (D-F), bitter 
292

293 295

\begin{tabular}{lccccc}
\hline Days after harvest & 0 & 0 to 35 & 35 & 35 to 63 & 63 \\
\hline Factor & PR & PP (\%) & PR & PP (\%) & PR \\
\hline $\begin{array}{l}\text { Healthy apple without bruise* } \\
\text { Healthy apple with bruise }\end{array}$ & 0.000 & 0 & 0.000 & 0 & 0.000 \\
$\begin{array}{l}\text { Bitter pit apple with mild } \\
\text { lesions }\end{array}$ & 0.001 & 1915 & 0.024 & 139 & 0.058 \\
$\begin{array}{l}\text { Bitter pit apple with severe } \\
\text { lesions }\end{array}$ & 0.083 & 202 & 0.054 & 45 & 0.078 \\
\hline
\end{tabular}

and 63 days after harvest. Note that the image registration slightly differs due to minor differences in fruit orientation during transportation between different days after harvest.

Table 1. Pit ratio and pit progression of representative healthy and bitter pit samples shown in Fig. 4.

${ }^{*}$ The PR and PP values were zero till $4^{\text {th }}$ or $5^{\text {th }}$ decimal place.

\subsection{Bitter Pit Distribution Evaluation}

Using CT image analysis of healthy and bitter pit affected fruits, it was also possible to determine the bitter pit distribution from top to bottom of an individual fruit and collectively quantify the disorder progression. Figure 5 shows the data for all inspected DAH.

More than $85 \%$ of the pits in the samples from field site- 1 (Fig. $5 \mathrm{~A}$ ) were located in the calyx-end of the fruit (section 3). In addition, 10 to $15 \%$ of pits were found in the middle (section 2) of the apple, and less than $3 \%$ of the pits were found at the stem-end (section 1) of the fruit. In field site-2 (Fig. 5B), the corresponding pit distribution in sections 3, 2, and 1 were about $54 \%, 30 \%$, and $10 \%$ in the sampled fruits. These findings are in accordance with prior studies that also reported that most of the bitter pit disorder symptoms are located in the fruit calyx end (Ferguson and Watkins, 1996; Nicolaï et al., 2006). This scenario corresponds to translocation of calcium inside fruits; however, more research is needed to confirm this aspect. 

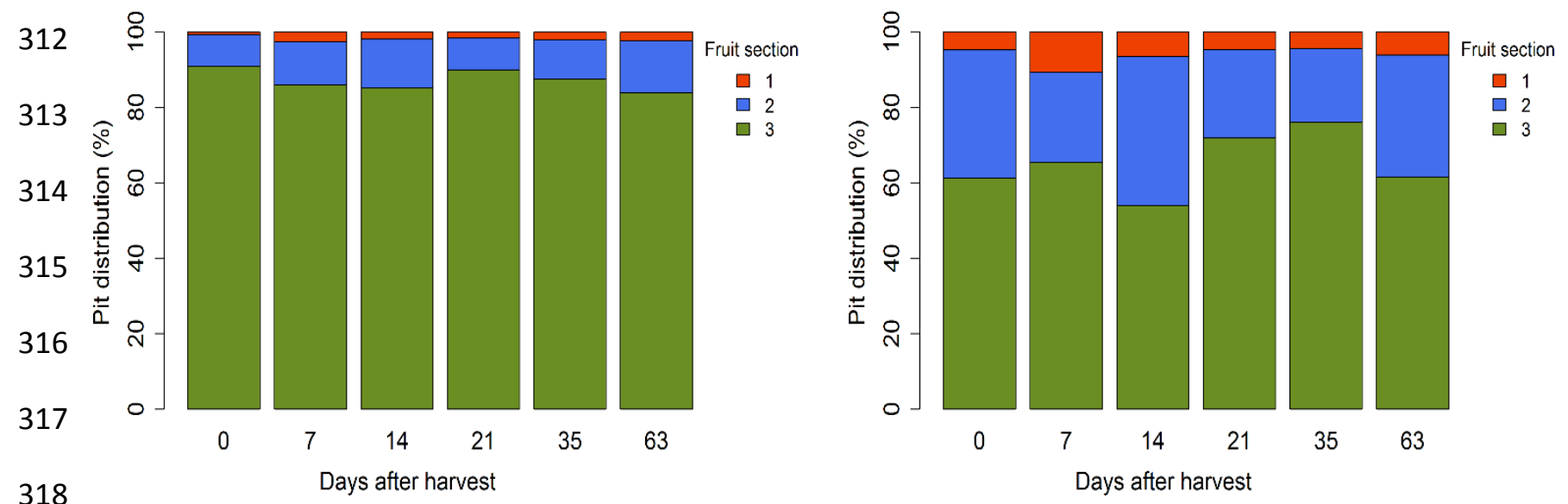

Fig. 5. Bitter pit distribution within sections of the fruit and progression during 63 day storage for samples from field sites-1 (A) and -2 (B). The section 1, 2 and 3 refers to stem-end, middle, and calyx-end of apples, respectively.

\subsection{Healthy and Bitter Pit Fruit Classification}

Figure 6 presents the curve describing the logistic regression model used to classify healthy and bitter pit samples based on pit ratio values. In the logistic regression based classification model, threshold of 0.5 was considered as the odds of apple being healthy or bitter

327 pit with corresponding pit ratio of 0.001 . This threshold was applied to all of the data in each 328 field site and the accuracies are reported in Table 2. Overall, the classification accuracies in detecting healthy and bitter pit fruits during different days of the storage ranged from $93-100 \%$ in field site-1. In field site-1, combining the six datasets resulted in $96 \%$ overall classification

331 accuracy with false positive and false negative of $2 \%$ and $7 \%$, respectively. However, overall 332 classification accuracy (70\%), false positive (38\%), and false negative (21\%) were much lower 333 in field site-2, when the datasets were combined. The key reason for large misclassification 334 error in field site-2 datasets was the bruising and decay on healthy apples that progressed 335 significantly through 63 days of storage. In future studies, shape features during image 336 processing will be used to detect specific defects. 


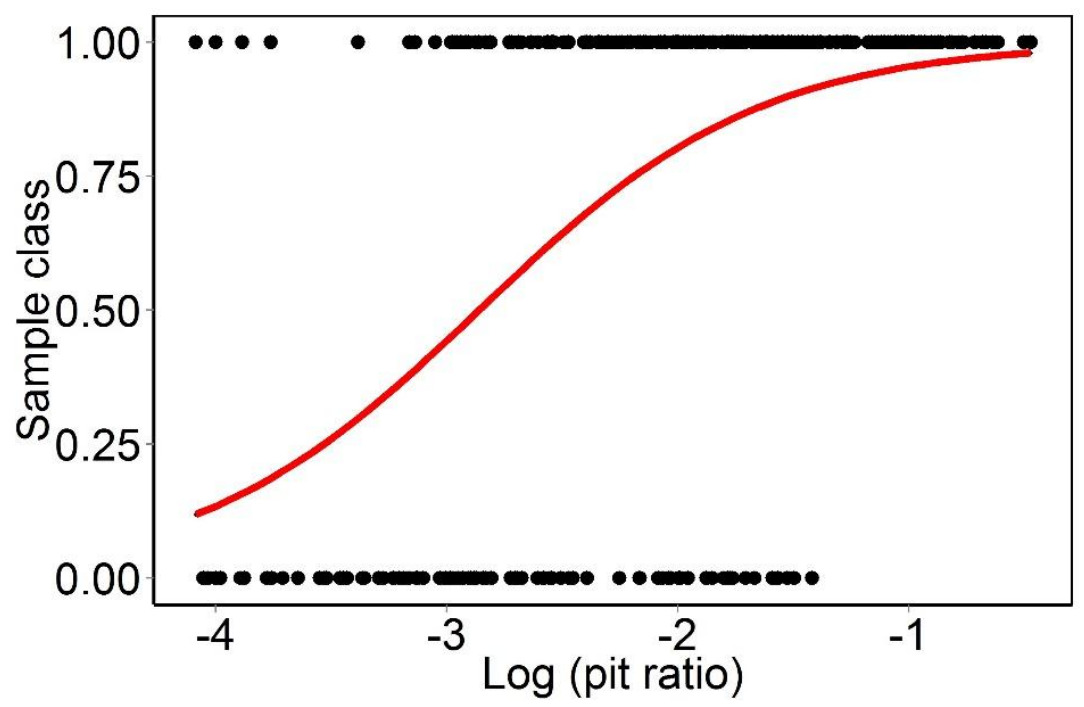

338 Fig. 6. Logistic regression between Pit Ratio and apple class ( 0 and 1 represent healthy and 339 bitter pit apples, respectively)

341 Table 2. Classification of healthy and bitter pit apples for 0, 7, 14, 21, 35 and 63 days after 342 harvest datasets.

\begin{tabular}{ccccccc}
\hline & \multicolumn{5}{c}{ Classification accuracy (\%) } \\
\cline { 2 - 6 } Days after Harvest & \multicolumn{3}{c}{ Field site-1 } & \multicolumn{3}{c}{ Field site-2 } \\
\cline { 2 - 6 } & Healthy & Bitter pit & Overall & Healthy & Bitter pit & Overall \\
\hline 0 & 95 & 90 & 93 & 90 & 70 & 80 \\
7 & 100 & 95 & 98 & 75 & 70 & 73 \\
14 & 100 & 90 & 95 & 55 & 70 & 63 \\
21 & 100 & 100 & 95 & 55 & 95 & 75 \\
35 & 100 & 100 & 100 & 50 & 85 & 68 \\
63 & 95 & 95 & 95 & 45 & 85 & 65 \\
Combined dataset & 98 & 93 & 96 & 62 & 78 & 70 \\
\hline
\end{tabular}

343

344

345

346

347

\section{Conclusions}

CT images of healthy and bitter pit affected apples were obtained and processed to determine the incidence of the physiological disorder in fruits. The images were processed and the overall bitter pit occurrence in each of healthy and bitter pit apples were evaluated as pit ratio at different periods during 63 days of storage. Pit distribution within bitter pit apples was 
also assessed. Results showed that the apples had major distribution of bitter pits in their calyxend. Moreover, classification of apples using pit ratio showed higher misclassification error in healthy apples with injury and bruises, especially in field site-2. For samples from field site-1, no

352 significant bruises or pits were developed in healthy apples. Both field sites showed increasing 353 bitter pits in bitter pit affected apples from $0.1 \mathrm{x}$ in slightly bitter pitted apples to $7 \mathrm{x}$ in severely

354 bitter pitted ones. CT technique demonstrated to be a useful tool in determining internal 355 incidence and progression of bitter pit disorder. The analysis of CT images can be applied to 356 detect other internal or external damages such as bruise and watercore. It is important to detect 357 bitter pit non-destructively in the early stages of the fruit storage period. Future work for this 358 research is to determine the potential of CT-based imaging to detect bitter pit in early stages of 359 pit development (pre-harvest) when visual symptoms are completely absent.

\section{Acknowledgements}

362 We are grateful to Dr. Tom Wilkinson from WSU Veterinary Clinical Sciences and the Teaching 363 Hospital for his assistance in completion of the study. This activity was funded by Washington 364 State Department of Agriculture (WSDA) Specialty Crop Block Grant Program (SCBGP). In 365 addition, the activity was partly supported by the USDA National Institute for Food and 366 Agriculture Hatch Projects WNP00821 and WNP00745. We would also like to thank Dr. 367 Jianfeng Zhou, Mr. Haitham Y. Bahlol and Mr. Ming Li as well as Borton Fruit, especially Mr. 368 Sky Johnson, Mr. Dave Hovde, and Mr. Dan Bowton for their help during this study. 369

\section{References}

371 Amarante, C.V.T., A. Miqueloto, S.T. de Freitas, C.A. Steffens, J.P.G. Silveira, and T.R. Corrêa, 2013: Fruit sampling methods to quantify calcium and magnesium contents to predict bitter pit development in 'Fuji'apple: A multivariate approach. Scientia Horticulturae 157, 
Amarante, C.V.T., Chaves, D.V., and Ernani, P.R., 2006. Composic, ão mineral e severidade de bitter pit em mac, ãs 'Catarina'. Revista Brasileira de Fruticultura 28, 51-54.

Bennedsen, B., and D. Peterson, 2005: Performance of a system for apple surface defect identification in near-infrared images. Biosystems engineering 90, 419-431.

Donis-González, I.R., D.E. Guyer, D.W. Fulbright, and A. Pease, 2014: Postharvest noninvasive assessment of fresh chestnut (Castanea spp.) internal decay using computer tomography images. Postharvest Biology and Technology 94, 14-25.

Du, C.-J., and D.-W. Sun, 2004: Recent developments in the applications of image processing techniques for food quality evaluation. Trends in Food Science \& Technology 15, 230249.

Ferguson, I., and C. Watkins, 1989: Bitter pit in apple fruit. Horticultural Relayers, Volume 11, 289-355.

Fuller, M., 1979: Cell ultrastructure in apple fruits in relation to calcium concentration and fruit quality Symposium on Mineral Nutrition and Fruit Quality of Temperate Zone Fruit Trees 92, 51-56.

Gutiérrez, R., 2002: Efecto de la ubicación en altitud y latitud de huertos de manzanos cv. Braeburn y Red Chief sobre la madurez de los frutos, la incidencia de bitter pit y en la capacidad de predicción mediante la infiltración de frutos con sales de magnesio. Available at: http://dspace.utalca.cl/bitstream/1950/792/1/RGutierrez.pdf.

Herremans, E., A. Melado-Herreros, T. Defraeye, B. Verlinden, M. Hertog, P. Verboven, J. Val, M.E. Fernández-Valle, E. Bongaers, and P. Estrade, 2014: Comparison of X-ray CT and MRI of watercore disorder of different apple cultivars. Postharvest Biology and 
398 Jones, W., Brinner, J., Kupferman, E., and Xiao, C-.L. 2007. Quick identification guide to apple postharvest defects and disorders. Available at: http://entomology.tfrec.wsu.edu/Cullage Site/Cards.html.

401

402

403

404

405

406

407

408

409

410
Lei, L., Q. Zhang, and D. Huang, 2014: A review of imaging techniques for plant phenotyping. Sensors 14.11, 20078-20111.

Lötze, E., C. Huybrechts, A. Sadie, K.I. Theron, and R.M. Valcke, 2006: Fluorescence imaging as a non-destructive method for pre-harvest detection of bitter pit in apple fruit (Malus domestica Borkh.). Postharvest biology and technology 40, 287-294.

Magwaza, L.S., and U.L. Opara, 2014: Investigating non-destructive quantification and characterization of pomegranate fruit internal structure using X-ray computed tomography. Postharvest biology and technology 95, 1-6.

Nicolaï, B.M., E. Lötze, A. Peirs, N. Scheerlinck, and K.I. Theron, 2006: Non-destructive measurement of bitter pit in apple fruit using NIR hyperspectral imaging. Postharvest biology and technology 40, 1-6.

Peng, C.-Y.J., K.L. Lee, and G.M. Ingersoll, 2002: An introduction to logistic regression analysis and reporting. The Journal of Educational Research 96, 3-14.

Perring, M., 1968: Mineral composition of apples. VII.-The relationship between fruit composition and some storage disorders. Journal of the Science of Food and Agriculture 19, 186-192.

Rosenberger, D., J. Schupp, C. Watkins, K. lungerman, S. Hoying, D. Straub, and L. Cheng, 2001: Honeycrisp: promising profit maker or just another problem child. NY Fruit Quarterly 9, 9-13. 
420 Salmanizadeh, F., S. Nassiri, A. Jafari, and M. Bagheri, 2015: Volume Estimation of Two Local

421 Pomegranate Fruit (Punica granatum L.) Cultivars and Their Components Using Non-

422 Destructive X-Ray Computed Tomography Technique. International Journal of Food

$423 \quad$ Properties 18, 439-455.

424 Schatzki, T., R. Haff, R. Young, I. Can, L.-C. Le, and N. Toyofuku, 1997: Defect detection in 425 apples by means of X-ray imaging. Transactions of the ASAE 40, 1407-1415.

426 Trexler, J. C., and Travis, J. 1993. Nontraditional regression analyses. Ecology 74, 1629-1637.

427 U.S. Department of Agriculture-National Agricultural Statistics Service (USDA-NASS), 201, Vol.

428 2015. Available at: http://www.usda.gov/nass/PUBS/TODAYRPT/crop0815.pdf.

429 Van der Boon, J., 1980: Prediction and control of bitter pit in apples. I. Prediction based on 430 mineral leaf composition, cropping levels and summer temperatures. J. Hort. Sci 55, 307-312.

432 Xiao-bo, Z., Z. Jie-wen, L. Yanxiao, and M. Holmes, 2010: In-line detection of apple defects $433 \quad$ using three color cameras system. Computers and Electronics in Agriculture 70, 129434 134. 